\title{
MATRIX FACTORIZATION AND LIFTING
}

\author{
PALLE E. T. JORGENSEN AND MYUNG-SIN SONG
}

\begin{abstract}
As a result recent interdisciplinary work, the processing of signals (audio, still-images, etc), a number of powerful matrix operations have led to advances both in engineering applications, and in mathematics. Much of it is motivated by ideas from wavelet algorithms. The applications are convincing measured against other processing tools already available, for example better compression (details below). We develop a versatile theory of factorization for matrix functions. By a matrix valued function we mean a function of one or more complex variables taking values in the group $G L(n, \mathbb{C})$ of invertible $n$ by $n$ matrices. Starting with this generality, there is a variety of special cases, also of interest, for example, one variable, or restriction to the case $n=2$; or consideration of subgroups of $G L(n, \mathbb{C})$; or $S L(n, \mathbb{C})$, i.e., specializing to the case of determinant equal to one. We will prove a number of factorization theorems and sketch their application to signal (image processing) in the framework of multiple frequency bands.
\end{abstract}

\section{Contents}

1. Introduction

2. Matrix Valued Functions

3. Systems of Filters

3.1. Operations on Time-Signals

4. Groups of Matrix Functions

5. Group Actions

5.1. Factorizations

5.2. Notational Conventions

6. Divisibility and residues for matrix-functions 12

6.1. The $2 \times 2$ case

6.2. The $3 \times 3$ case

6.3. The $N \times N$ case 16

7. Quantization 18

$\begin{array}{ll}\text { References } & 21\end{array}$

\section{INTRODUCTION}

Starting with the early work on wavelets(the 1980ties), there is now an important body of theory at the crossroads of a number of mathematical areas (harmonic

Date: March, 2010.

1991 Mathematics Subject Classification. Primary 46M05, 47B10, 60H05, 62M15.

Key words and phrases. Hilbert space, Tensor Product, Trace-class, Spectral Theorem, Harmonic Analysis, Fractal Analysis, Karhunen-Loève Transforms, Stochastic Integral.

Work supported in part by the U.S. National Science Foundation. 
analysis and function theory) on the one side, and theoretical signal processing on the other. An especially convincing instance of this was the recent use of wavelet algorithms by the JPEG group. (The term "JPEG" is an acronym for the Joint Photographic Experts Group which created the standard.) The achieved compression resulting from these techniques is used, for example in a variety of image file formats. JPEG/Exif is the most common of them, used in digital cameras and other image devices. Moreover, these mathematical tools are now part of common formats for storing and transmitting photographic images on the web.

The marriage of the two subjects came from the early realization that filters generating the most successful wavelet bases could be obtained from an adaptation of more classical sub-band filtering operations used in signal-processing; with the notions of down-sampling and up-sampling being intrinsic to numerical wavelet algorithms used in for example compression of signals, and more generally of images. For a lucid account, see e.g., [Law99].

A common feature for the more traditional processing tools is the division into sub-bands, but in modern applications such a sub-division is more subtle. Here we develop and refine a procedure which uses factorization of families of matrixvalued function. These operations are done on the frequency-side; but it is fairly easy, at the end, to convert back to the time-signal itself. Here we use the concept "time-signal" widely allowing for systems of numbers indexed by pixels, such as grey-scale numbers for still-images; or, for color, more complicated configuration of pixel matrices.

To be successful, a signal processing must allow a practical procedure for breaking down an overall processing into the smallest ingredients. The role of factorization of matrix functions is precisely to accomplish this: In the case of two frequency bands, 2 by 2 matrix functions suffice, and in this case, the corresponding factorization (see e.g., Sweldens et al, [Swe96, Swe98] and [CC08]). In this case, the factorization goes by the name "lifting" and the product is a (perhaps) long string of upper and lower triangular matrices, alternation between upper and lower. But each of these basic factors will then just encode a function of the frequency variable, corresponding to a filtering step in the overall process.

Below we demonstrate how this is done in the case of a processing involving multiple bands, as well as the features dictated by modern applications.

\section{Matrix Valued Functions}

Matrix valued functions of one or more complex variable, taking values in the group $S L(2, \mathbb{C})$, have a number of uses in both pure and applied mathematics. Here we will focus on a framework in the signal processing literature called "the lifting scheme," or "lifting algorithms." A main result there (suitable restrictions) is the assertion that, in the case of polynomial entries, these matrix functions factor into finite products of alternating upper and lower diagonal matrix functions.

Even though pioneering ideas are from engineering, we hope to show that they are of interest in pure mathematics as well, especially in operator theory.

The result is of special practical significance in building filters with the use of two frequency bands with a recursive input-output model; using as input filtered signals from the low band, and producing an additive perturbation to the high frequency channel. This is continued recursively, with reversal of the role of high and low in each step. For some of the literature, we refer to [SBT03, CC08, HCXH08] and 
many papers in the engineering literature. Since ealry pioneering ideas by Wim Sweldens, e.g., [SR91], the subject has since branched off in a variety of directions both applied and pure; see [DS98] and the papers cited there.

One of our motivations here is the desire to extend and refine this method to the case of multiple bands. In the simplest case, by this we mean that signals are viewed as time function (discrete time) and each time-function generating a frequency response function (generating function) of a complex variable. In applications it is possible to encode time-signals or their generating functions as vectors in a Hilbert space $\mathcal{H}$. And to do this in such a way that a finite selection of frequency bands will then correspond to a system of closed subspaces in $\mathcal{H}$. A direct generalization of the case $n=2$ to $n>2$ is not feasible. We note that the factorization conclusion for $n=2$ into alternating products of upper and lower, does not carry over to $n>2$; but, motivated by applications, we outline a version that does.

A new element in our approach is the use of some operator theory initiated into the study of sub-band filtering in [BJ02a]; see also [BJ02b, JS09, Son06].

While the notion of upper/lower factorization is both versatile, and old, dating back to Gauss, it has a variety of modern incarnations, many of which are motivated by computation. On the pure side, we list the Iwasawa decomposition for semisimple Lie groups [Iwa49], and on the applied side, the matrix formulation of the algorithm of Gram and Schmidt for creating orthogonal vectors in complex Hilbert space [Akh65].

In the signal processing, the context is different: Here we deal with infinitedimensional groups of matrix functions; functions taking values in one of the finitedimensional Lie groups, different groups for different purposes.

Of the many presentations in the literature dealing with signal processing applications, the following papers are especially relevant to our present approach: [Law04, Law02, Law00, Law99, BR91, BRa, BRb] . Equally important are the papers [DS98, Swe98, Swe96]; as well as their presentation in the book [JlCH01] .

\section{Systems of Filters}

In this section we present the mathematics of some key concepts from signal processing. In their mathematical form, these ideas are timeless, and pretty versatile; thus applying equally well to signals of a more basic nature, as well as to signal processing in wireless communication. With suitable adaptation, these in fact are tools for image processing as well.

The purpose of our presentation here is to set up the problems for the framework of matrix analysis. By this we mean the study of functions in one or several complex variables, but taking values in a particular Lie group of invertible matrices, for example the general linear group $G L_{N}$, the group $\mathcal{U}_{N}$ of unitary $N$ by $N$ matrices, or one of the symplectic groups, etc. The choice of group in our analysis depends on the problem at hand. While the Lie groups $G$ in the above list are finitedimensional, the moment we pass to the group of functions taking values in $G$, this will be an infinite-dimensional group.

\section{Setting.}

- $\mathbb{C}$ : the complex plane

- $D:=\{z \in \mathbb{C} ;|z|<1\}$

- $\partial D:=\mathbb{T}=\{z \in \mathbb{C} ;|z|=1\}=\left\{e^{i \theta} ; \theta \in \mathbb{R}\right.$, or $\left.\mathbb{R} / 2 \pi \mathbb{Z}\right\}$ 
- Let $\Omega \subset \mathbb{C}$ be an open subset such that $\mathbb{T} \subset \Omega$. Algebras of functions and Fourier representation:

$$
f(z)=\sum_{k} a_{k} z^{k}=\sum_{k} a_{k} e^{i 2 \pi k \theta} .
$$

If $g(z)=\sum_{k} b_{k} z^{k}$, we shall impose conditions at $k \rightarrow \infty$ such that

$$
\begin{aligned}
f(z) g(z) & =\sum_{n} c_{n} z^{n}, \quad \text { where } \\
c_{n} & =\sum_{k} a_{k} b_{n-k}
\end{aligned}
$$

can be justified.

\subsection{Operations on Time-Signals.}

Filtering If $\left(b_{m}\right)_{m \in \mathbb{Z}}$ is a time-signal, we say that $(3.2)$ is a filter acting on $\left(b_{m}\right)$.

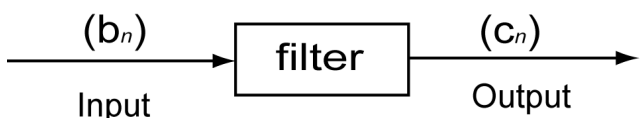

Below we will be using the notations $\uparrow$ and $\downarrow$ to denote operators, i.e., transformations acting on spaces of signals.

Upsampling $\uparrow$ Fix $N \in \mathbb{Z}_{+}, N>1$. Consider a time-signal $\left(b_{k}\right)$ and a frequency response function $g(z)=\sum_{k} b_{k} z^{k}$.

Action on the signal: $\left(b_{k}\right) \mapsto\left(c_{n}\right)$ where

$$
c_{n}= \begin{cases}b_{k} & \text { if } N \mid n, \text { i.e., } \exists k \text { such that } n=N \cdot k . \\ 0 & \text { if } N \nmid n .\end{cases}
$$

Action on the functions:

$$
g(z) \mapsto h(z) \text {, where } h(z)=g\left(z^{N}\right) .
$$

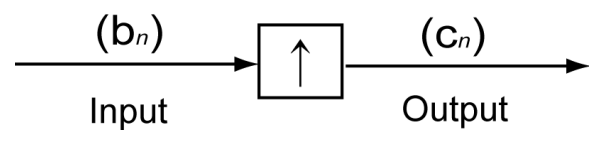

Downsampling $\downarrow$ Fix $N \in \mathbb{Z}_{+}, N>1$. Consider a time-signal $\left(b_{k}\right)$ and a frequency response function $g(z)=\sum_{k} b_{k} z^{k}$.

Action on Signal: $\left(b_{k}\right) \mapsto\left(c_{n}\right)$ where

$c_{n}=b_{n N} \quad$ for all $n \in \mathbb{Z} \quad$ (i.e., discarting input $b_{k}$ when $k$ is not divisible by $N$.) Action on functions: $g(z) \mapsto h(z)$, where

$$
h(z)=\frac{1}{N} \sum_{w \in \mathbb{T}, \quad w^{N}=z} g(w), \quad \text { average over } N^{t h} \text { roots. }
$$




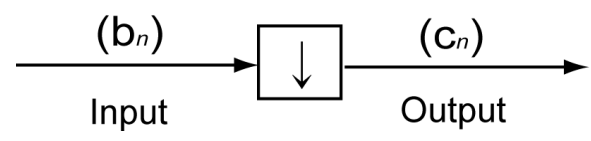

Frequency bands: We say that a partition of $-\pi \leq \theta<\pi$ into $N$ sub-intervals.

$$
\frac{2 \pi k}{N}-\frac{\pi}{N} \leq \theta<\frac{2 \pi k}{N}+\frac{\pi}{N}
$$

is a sub-band partition with $k=0$ corresponding to the lowest band, and $k=\left[\frac{N}{2}\right]$ the highest band.

Definition 3.1. Let $N \in \mathbb{Z}_{+}$be given, and set $\zeta_{N}:=e^{i \frac{2 \pi}{N}}=$ the principal $N^{t h}$ root of 1 . Set

$$
\left(A_{N} g\right)(z):=\frac{1}{N} \sum_{k=0}^{N-1} g\left(\zeta_{N}^{k} z\right)
$$

Note the summation in (3.7) is over the cyclic group $\mathbb{Z}_{N}=\mathbb{Z} / N \mathbb{Z}(=\{0,1, \cdots, N-$ $1\})$.

\section{Lemma 3.2.}

$$
A_{N}=\uparrow \downarrow \quad \text { (downsampling followed by upsampling), }
$$

i.e., composition of operators.

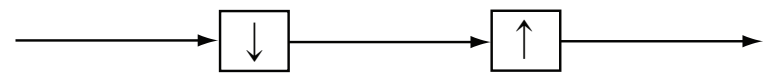

Proof.

$$
\begin{aligned}
\left(A_{N} g\right)(z) & =(\downarrow g)\left(z^{N}\right) \\
& =\frac{1}{N} \sum_{w \in \mathbb{T},} g(w) \\
& =\frac{1}{N} \sum_{k \in \mathbb{Z}_{N}=z^{N}} g\left(\zeta_{N}^{k} z\right), \quad \text { summation over the cyclic group of order } N,
\end{aligned}
$$

which is the formula in (3.7).

Corollary 3.3. The action on $A_{N}$ on time-signal $\left(b_{k}\right)$ is as follows

$$
\left(A_{N} b\right)_{k}= \begin{cases}b_{k} & \text { if } N \mid k, \\ 0 & \text { if } N \nmid k .\end{cases}
$$

Definition 3.4. Let $N \in \mathbb{Z}_{+}$be given; the two systems of functions

$$
F=\left(f_{k}\right)_{k \in \mathbb{Z}_{N}} \text { and } G=\left(g_{k}\right)_{k \in \mathbb{Z}_{N}}
$$

is said to be a perfect reconstruction filter iff

$$
\sum_{k \in \mathbb{Z}_{N}} M_{g_{k}} A_{N} M_{f_{k}}=I \quad \text { (see Fig. 1) }
$$

where the operator $I$ on the RHS in (3.9) is the identity operator.

In the engineering lingo, e.g. (3.9) is expressed as follows: 


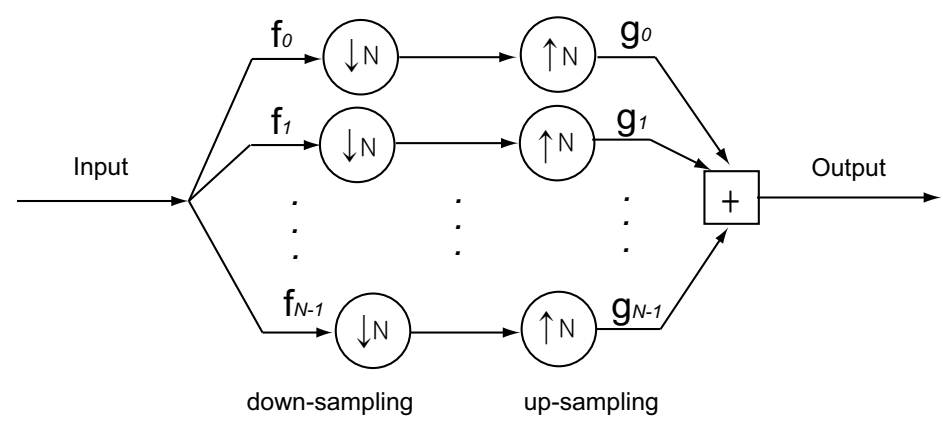

FiguRe 1. Perfect reconstruction in a subband filtering as used in signal and image processing.

Definition 3.5. For such function $f(z)=\sum_{n \in \mathbb{Z}} a_{n} z^{n}$, set

$$
f^{*}(z)=\sum_{n \in \mathbb{Z}} \overline{a_{-n}} z^{n} .
$$

\section{Groups of Matrix Functions}

The groups of functions taking values in a particular Lie group $G$ (see section 2 for details) act naturally on vector valued functions. This action is simply pointwise: If $G$ is a group of $N$ by $N$ complex matrices, the action will then be on functions mapping into $\mathbb{C}^{N}$, i.e., complex $N$-space. This is important as the mathematics of filters in signal processing takes place on $\mathbb{C}^{N}$-valued functions. The way this is done is outlined below; keeping in mind our framework of factorization for a particular (infinite-dimensional) group of functions taking values in some Lie group $G$.

Definition 4.1. A system $F=\left(f_{k}\right)_{k \in \mathbb{Z}_{N}}$ is said to be an orthogonal filter (with $N$ bands) iff (3.9) holds with $g_{k}=f_{k}^{*}$.

Proposition 4.2. A system $F=\left(f_{k}\right)_{k \in \mathbb{Z}_{N}}$ is an orthogonal filter with $N$ bands iff the $N \times N$ matrix

$$
U_{F}(z):=\left(f_{j}\left(\zeta_{N}^{k} z\right)\right)_{(j, k) \in \mathbb{Z}_{N} \times \mathbb{Z}_{N}}
$$

is unitary for all $z \in \mathbb{T}(=\partial D)$ ).

Proof. An application of the previous lemma.

Definition 4.3. An $N \times N$ matrix-valued function $U$ is said to be unitary iff $U(z)$ is a unitary matrix for all $z \in \mathbb{T}$.

Let the set of all orthogonal $N$-filters be denoted $\mathcal{O} \mathcal{F}_{N}$, and the set, all unitary matrix functions $\mathcal{U} \mathcal{M}_{N}$.

Definition 4.4. Let $U$ be an $N \times N$ matrix-function, and let $F=\left(f_{k}\right)_{k \in \mathbb{Z}_{N}}$ be a function system. Set

$$
G(z):=U\left(z^{N}\right) F(z)
$$

or equivalently

$$
g_{k}(z)=\sum_{j \in \mathbb{Z}_{N}} U_{k, j}\left(z^{N}\right) f_{j}(z) .
$$


Lemma 4.5. With the action (4.2), the group $\mathcal{U} \mathcal{M}_{F}$ acts transitively on $\mathcal{O} \mathcal{F}_{N}$.

Proof. If $F \in \mathcal{O} \mathcal{F}_{N}$, and $U \in \mathcal{U} \mathcal{M}_{F}$, then the action (4.2) is easily seen to make $G(z)=U\left(z^{N}\right) F(z)$ an orthogonal filter.

Let $F$ and $G$ be in $\mathcal{O} \mathcal{F}_{N}$, and set

$$
U_{j, k}(z)=\frac{1}{N} \sum_{w \in \mathbb{T}, \quad w^{N}=z} g_{k}(w) \overline{f_{j}(w)} .
$$

An inspection shows that $U=\left(U_{j, k}\right)$ is in $\mathcal{U} \mathcal{M}_{F}$, and that (4.3) is satisfied.

Corollary 4.6. Let $N \in \mathbb{Z}_{+}$be given. Set

$$
\begin{aligned}
& b(z)=\left[\begin{array}{c}
1 \\
z \\
z^{2} \\
\vdots \\
z^{N-1}
\end{array}\right] ; \text { then } \\
& \mathcal{O F}_{N}=\mathcal{U M}_{F} b \\
& =:\left\{U\left(z^{N}\right) b(z) ; U \in \mathcal{U M}_{F}\right\} .
\end{aligned}
$$

Definition 4.7. Let $N \in \mathbb{Z}_{+}$be given, and let $\langle\cdot, \cdot\rangle_{N}$ be the usual inner product in $\mathbb{C}^{N}$, i.e.,

$$
\langle v, w\rangle_{N}:=\sum_{k} \overline{v_{k}} w_{k} .
$$

If $F$ and $G$ are $\mathbb{C}^{N}$-valued matrix, functions, set

$$
\ll F, G \gg_{N}(z)=\frac{1}{N} \sum_{w \in \mathbb{T},}\langle F(w), G(w)\rangle_{N}=\downarrow\langle F, G\rangle_{N}(z)
$$

Lemma 4.8. Let $N \in \mathbb{Z}_{+}$be fixed; and let $A$ and $B$ be matrix functions. Then

$$
\ll A b, B b \gg_{N}=\operatorname{trace}\left(A^{*}(z) B(z)\right),
$$

where $b$ is given by (4.5).

Proof.

$$
\begin{aligned}
\ll A b, B b \gg_{N} & =\frac{1}{N} \sum_{w^{N}=z} \sum_{j} \sum_{k} \sum_{l} \overline{A_{j, k}(z) w^{k}} B_{j, l}(z) w^{l} \\
& =\sum_{j} \sum_{k} \sum_{l} \overline{A_{j, k}(z)} B_{j, l}(z) \frac{1}{N} \sum_{w^{N}=z} \overline{w^{k}} w^{l} \\
& =\sum_{j} \sum_{k} \sum_{l} \overline{A_{j, k}(z)} B_{j, l}(z) \delta_{k, l} \\
& =\sum_{j} \sum_{k} \overline{A_{j, k}(z)} B_{j, l}(z) \\
& =\operatorname{trace}\left(A(z)^{*} B(z)\right),
\end{aligned}
$$

which is the desired conclusion. 
Definition 4.9. Let $\mathcal{H}=L^{2}(\mathbb{T})$ be the Hilbert space given by

$$
\frac{1}{2 \pi} \int_{-\pi}^{\pi}\left|\varphi\left(e^{i \theta}\right)\right|^{2} d \theta=\sum_{n \in \mathbb{Z}}\left|b_{n}\right|^{2}
$$

where $\varphi\left(e^{i \theta}\right)=\sum_{n \in \mathbb{Z}} b_{n} e^{i n \theta}$ is the Fourier representation.

Let $F=\left(f_{j}\right)_{j \in \mathbb{Z}_{N}}$ be a function system on set

$$
\left(S_{j} \varphi\right)(z)=f_{j}(z) \varphi\left(z^{N}\right) .
$$

Lemma 4.10. Let $N \in \mathbb{Z}_{+}$be given, and let $F=\left(f_{j}\right)_{j \in \mathbb{Z}_{+}}$be a function system. Then $F \in \mathcal{O} \mathcal{F}_{N}$ if and only if the operators $S_{j}$ in (4.10) satisfy

$$
\begin{aligned}
& S_{j}^{*} S_{k}=\delta_{j, k} I \\
& \sum_{j \in \mathbb{Z}_{N}} S_{j} S_{j}^{*}=I
\end{aligned}
$$

where $I$ denotes the identity operator in $\mathcal{H}=L^{2}(\mathbb{T})$; compare with Fig. 1.

Proof. This is a direct application of the two previous lemmas.

\section{Group Actions}

In this section we state our first results regarding factorization in (infinitedimensional) groups of functions taking values in some Lie group $G$; matrix-functions for short.

We outline notational conventions and state the factorization problem in a simple case. Generalities will be added later. We begin with two key lemmas to be applied later.

Let $N \in \mathbb{Z}_{+}$be given $(N>1)$, and consider $F=\left(f_{j}\right)_{j \in \mathbb{Z}_{+}}$in $\mathcal{F}_{2}(N):=$ $L^{2}\left(\mathbb{T}, \mathbb{C}^{N}\right)=\sum_{0}^{N-1 \oplus} L^{2}(\mathbb{T})$ with

$$
\|F\|_{2}^{2}=\sum_{j=0}^{N-1}\left\|f_{j}\right\|_{L^{2}(\mathbb{T})}^{2}<\infty .
$$

We will be making use of the special vector $b \in \mathcal{F}_{2}(N)$,

$$
b(z)=\left[\begin{array}{c}
1 \\
z \\
z^{2} \\
\vdots \\
z^{N-1}
\end{array}\right]
$$

see Corollary 4.6.

Let

$$
\left(S_{j} f\right)(z)=z^{j} f\left(z^{N}\right)
$$

be the Cuntz-representation from Definition 4.9 and Lemma 4.10.

Lemma 5.1. Let $N \in \mathbb{Z}_{+}$be fixed, $N>1$, and let $A=\left(A_{j, k}\right)$ be an $N \times N$ matrixfunction with $A_{j, k} \in L^{2}(\mathbb{T})$. Then the following two conditions are equivalent:

(i) For $F=\left(f_{j}\right) \in \mathcal{F}_{2}(N)$, we have $F(z)=A\left(z^{N}\right) b(z)$.

(ii) $A_{i, j}=S_{j}^{*} f_{i}$ where the operators $S_{i}$ are from the Cuntz-relations (5.1). 
Proof. (i) $\Rightarrow$ (ii). Writing out the matrix-operation in (i), we get

$$
f_{i}(z)=\sum_{j} A_{i, j}\left(z^{N}\right) z^{j}=\sum_{j}\left(S_{j} A_{i, j}\right)(z)
$$

Using $S_{j}^{*} S_{k}=\delta_{j, k} I$, we get $A_{i, j}=S_{j}^{*} f_{i}$ which is (ii).

Conversely, assuming (ii) and using $\sum_{j} S_{i} S_{j}^{*}=I$, we get $\sum_{j} S_{j} A_{i, j}=f_{i}$ which is equivalent to (i) by the computation in (5.2) above.

Corollary 5.2. Let $N \in \mathbb{Z}_{+}$be fixed, and let $A$ and $B$ be $N \times N$ matrix-functions with $L^{2}$-entries. Then the following are equivalent:

(i) $A\left(z^{N}\right) b(z)=B\left(z^{N}\right) b(z)$ and

(ii) $A \equiv B$.

5.1. Factorizations. We will now sketch the first step in the general conclusions about factorization.

In the arguments below, the size of the problem has two parts:

(a) The matrix size, i.e., the size of $N$ where we consider $N \times N$ matrices.

(b) The number of factors in our factorizations.

To illustrate the idea, we begin with consideration of the case when both numbers in (a) and (b) are 2.

Lemma 5.3. Let

$$
A=\left(\begin{array}{ll}
A & B \\
C & D
\end{array}\right)
$$

be a $2 \times 2$ matrix-function, and let

$$
\left\{\begin{array}{l}
f_{0}(z)=A\left(z^{2}\right)+z B\left(z^{2}\right) \\
f_{1}(z)=C\left(z^{2}\right)+z D\left(z^{2}\right)
\end{array}\right.
$$

Let $L$ and $U$ be scalar functions. Then the following are equivalent:

(i)

$$
\left(\begin{array}{ll}
1 & 0 \\
L & 1
\end{array}\right)\left(\begin{array}{ll}
1 & U \\
0 & 1
\end{array}\right)=\left(\begin{array}{ll}
A & B \\
C & D
\end{array}\right)
$$

(ii) $U=S_{1}^{*} f_{0}$ and $L=S_{0}^{*} f_{1}$.

Proof. This is a direct consequence of the lemmas in section 4 .

\subsection{Notational Conventions.}

(i) Let $N \in \mathbb{Z}_{+}$be fixed. We will denote $N \times N$ matrix function $A(z)=$ $\left(A_{j, k}(z)\right)_{j, k \in \mathbb{Z}_{N}}$ with row/column indices from $\{0,1, \cdots, N-1\}$, and $N$-column vector functions

$$
v(z)=\left[\begin{array}{c}
v_{0}(z) \\
v_{1}(z) \\
\vdots \\
v_{N-1}(z)
\end{array}\right]
$$

We will consider $A$ acting on the vector $v$ as follows:

$$
A_{N}[v](z):=A\left(z^{N}\right) v(z)
$$

where the RHS in (5.3) is a $(N \times N)(N \times 1)$ matrix-product. Note the subscript $N$ in the definition (5.3) above. 
(ii) If $f$ and $g$ are two scalar valued functions, we set

$$
\langle f, g\rangle_{N}(z)=\frac{1}{N} \sum_{w \in \mathbb{T}} \overline{w^{N}=z}(w) g(w),
$$

i.e., this is an inner product taken values in spaces of funtions.

(iii) If $f$ is given, we set

$$
\left(S_{f} \varphi\right)(z):=f(z) \varphi\left(z^{N}\right)
$$

and

$$
\left(S_{f}^{*} \varphi\right)(z):=\frac{1}{N} \sum_{w \in \mathbb{T}} \overline{w^{N}=z}\left(\overline{f(w)} \varphi(w)=\langle f, \varphi\rangle_{N}(z)\right.
$$

(iv) Note that $S_{f}^{*}$ is the $L^{2}(\mathbb{T})$-adjoint operator, i.e., if $\varphi, \psi \in L^{2}(\mathbb{T})$, then

$$
\left\langle S_{f} \varphi, \psi\right\rangle_{L^{2}(\mathbb{T})}=\left\langle\varphi, S_{f}^{*} \psi\right\rangle_{L^{2}(\mathbb{T})}
$$

where $\langle\cdot, \cdot\rangle_{L^{2}(\mathbb{T})}$ denotes the usual inner product in the Hilbert space $L^{2}(\mathbb{T})$.

Lemma 5.4. Let $f_{0}, f_{1}, \cdots, f_{N-1}$ be a system of $N$ complex functions. (For the present purpose, we only need to assume that each $f_{j}$ is in $L^{\infty}(\mathbb{T})$.)

Then the following three conditions are equivalent:

(i) The functions $f_{j}$ satisfy

$$
\left\langle f_{j}, f_{k}\right\rangle_{N}(z)=\delta_{j, k} 1, \quad \forall z \in \mathbb{T}, \quad \text { module-orthogonality. }
$$

(ii) The operator $S_{f_{j}}$ satisfy the Cuntz-relations

$$
\left\{\begin{array}{l}
S_{f_{j}}^{*} S_{f_{k}}=\delta_{j, k} I_{L^{2}(\mathbb{T})}, \quad \text { and } \\
\sum_{j=0}^{N-1} S_{f_{j}} S_{f_{j}}^{*}=I_{L^{2}(\mathbb{T})}
\end{array}\right.
$$

(iii) With $\zeta_{N}:=e^{i \frac{2 \pi}{N}}$, form the matrix function

$$
M_{N}(z)=\left(f_{j}\left(\zeta_{N}^{k} z\right)\right)_{j, k \in \mathbb{Z}_{N}} .
$$

Then $M_{N}$ is a unitary matrix-function.

Definition 5.5. A system of function $\left(f_{j}\right)_{j \in \mathbb{Z}_{N}}$ satisfying any one of the three conditions in Lemma 5.4 is called an orthogonal system of sub-band filters.

Remark 5.6. An advantage of the operator formalism in Lemma 5.4 (representations of Cuntz algebras) is that the operators $P_{j}:=S_{j} S_{j}^{*}$ will then be a system of mutually orthogonal projections, projections onto the subspaces in $L^{2}(\mathbb{T}) \sim l^{2}(\mathbb{Z})$ corresponding to frequency bands, with $P_{0}=$ projection onto the subspace of the lowest band.

This simplifies in the case of just two bands: then the family

$$
Q_{i}:=S_{1}^{i} S_{0} S_{0}^{*} S_{1}^{*^{i}}, \quad i=0,1,2, \cdots
$$

is infinite and mutually orthogonal. We get a well-defined infinite sum (of orthogonal projections):

$$
\sum_{i=0}^{\infty} Q_{i}=I_{L^{2}(\mathbb{T})} \sim I_{l^{2}} .
$$


To justify (5.11), we use that $\lim _{n \rightarrow \infty} S_{1}^{n} S_{1}^{*^{n}}=0$ holds in the strong operator topology. With this, we then get a useful version of the pyramid algorithm, and even an image-subdivision scheme; see Fig 3 and Fig 6.

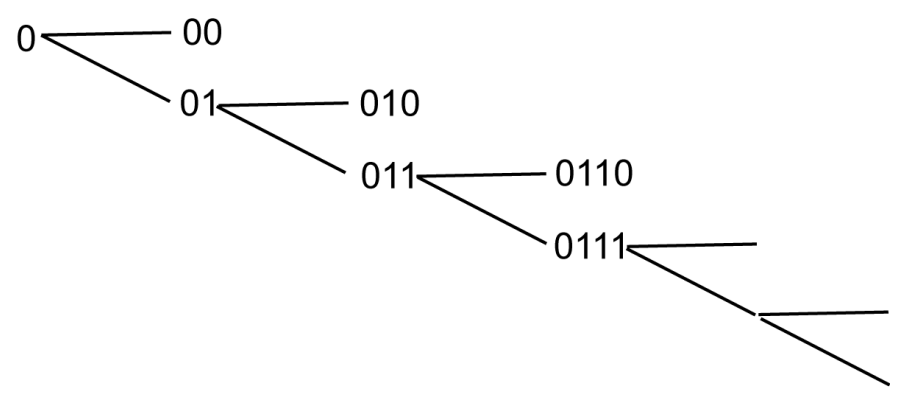

FiguRE 2. Pyramid Algorithm.

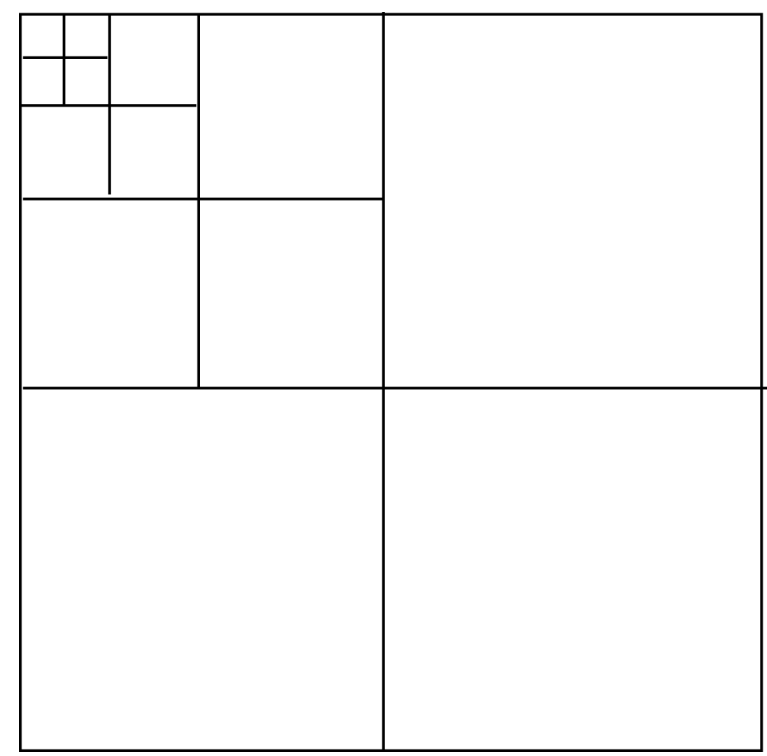

Figure 3. For images with $N=4$.

Corollary 5.7. Every orthogonal system of sub-band filters $F=\left[f_{j}\right]_{j \in \mathbb{Z}_{N}}$ has the form

$$
F=U_{N}[b]
$$

where $U$ is a unitary matrix-function, and where

$$
b=\left(\begin{array}{c}
1 \\
z \\
z^{2} \\
\vdots \\
z^{N-1}
\end{array}\right)
$$


and where $U_{N}[b](z)=U\left(z^{N}\right) b(z)$.

Definition 5.8. A matrix-function or a vector function is said to be of polynomial type, or a polynomial matrix-function, if its entries are polynomials: If $H \subset \mathbb{Z}$ is a finite subset of the integers and $a: H \mapsto \mathbb{C}$ is a function on $H$, by a polynomial we shall mean the expression

$$
f_{H}(z):=\sum_{n \in H} a_{n} z^{n}
$$

so a finite Laurent expression.

The difference $D=\max H-\min H$ will be called the degree of $f_{H}$.

Let $N \in \mathbb{Z}_{+}$be given and fixed. The following terminology will be used:

$G L_{N}(\mathrm{pol})$ : the $N \times N$ polynomial matrix function $A$ such that $A^{-1}$ is also polynomial.

$$
S L_{N}(\mathrm{pol}):=\left\{A \in G L_{N}(\mathrm{pol}) ; \quad \operatorname{det} A \equiv 1\right\} .
$$

Our work in matrix function give the following:

Theorem 5.9. (Sweldens [SR91]) Let $A \in S L_{2}$ (pol), then there are $l, p \in \mathbb{Z}_{+}$, $K \in \mathbb{C} \backslash\{0\}$, and polynomial functions $U_{1}, \ldots, U_{p}, L_{1}, \ldots, L_{p}$ such that

$$
A(z)=z^{l}\left(\begin{array}{cc}
K & 0 \\
0 & K^{-1}
\end{array}\right)\left(\begin{array}{cc}
1 & U_{1}(z) \\
0 & 1
\end{array}\right)\left(\begin{array}{cc}
1 & 0 \\
L_{1}(z) & 1
\end{array}\right) \cdots\left(\begin{array}{cc}
1 & U_{p}(z) \\
0 & 1
\end{array}\right)\left(\begin{array}{cc}
1 & 0 \\
L_{p}(z) & 1
\end{array}\right) .
$$

Remark 5.10. Note that if

$$
\left(\begin{array}{ll}
\alpha & \beta \\
\gamma & \delta
\end{array}\right) \in S L_{2}(\mathrm{pol})
$$

then one of the two functions $\alpha(z)$ or $\delta(z)$ must be a monomial.

\section{DivisibILITY AND RESIDUES FOR MATRIX-FUnCTIONS}

The present section deals with some key steps in the proof of our two main theorems.

6.1. The $2 \times 2$ case. To highlight the general ideas, we begin with some details worked out in the $2 \times 2$ case; see equation (5.15).

First note that from the setting in Theorem 5.9, we may assume that matrix entries have the form $f_{H}(z)$ as in (5.13) but with $H \subset\{0,1,2, \cdots\}$, i.e., $f_{H}(z)=$ $a_{0}+a_{1} z+\cdots$. This facilitates our use of the Euclidean algorithm.

Specifically, if $f$ and $g$ are polynomials (i.e., $H \subset\{0,1,2, \cdots\}$ ) and if $\operatorname{deg}(g) \leq$ $\operatorname{deg}(f)$, the Euclidean algorithm yields

$$
f(z)=g(z) q(z)+r(z)
$$

with $\operatorname{deg}(r)<\operatorname{deg}(g)$. We shall write

$$
q=q u o t(g, f), \quad \text { and } \quad r=\operatorname{rem}(g, f) .
$$

Since

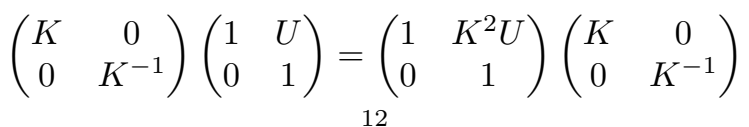


we may assume that the factor

$$
\left(\begin{array}{cc}
K & 0 \\
0 & K^{-1}
\end{array}\right)
$$

from the equation (5.15) factorization occurs on the rightmost place.

We now proceed to determining the polynomials $U_{1}(z), L_{1}(z), \cdots$, etc. inductively starting with

$$
A=\left(\begin{array}{cc}
1 & U \\
0 & 1
\end{array}\right) B
$$

where $U$ and $B$ are to be determined. Introducing (5.12), this reads

$$
A\left(z^{2}\right)\left(\begin{array}{l}
1 \\
z
\end{array}\right)=\left(\begin{array}{cc}
1 & U\left(z^{2}\right) \\
0 & 1
\end{array}\right) B\left(z^{2}\right)\left(\begin{array}{l}
1 \\
z
\end{array}\right)=\left(\begin{array}{cc}
1 & U\left(z^{2}\right) \\
0 & 1
\end{array}\right)\left(\begin{array}{l}
h(z) \\
k(z)
\end{array}\right)
$$

But the matrix function

$$
A=\left(\begin{array}{ll}
\alpha & \beta \\
\gamma & \delta
\end{array}\right)
$$

is given, and fixed see Remark 5.10. Hence

$$
\gamma\left(z^{2}\right)+\delta\left(z^{2}\right) z=k(z)
$$

is also fixed. The two polynomials to be determined are $u$ and $h$ in (6.4). Carrying out the matrix product in (6.4) yields:

$$
\alpha\left(z^{2}\right)+\beta\left(z^{2}\right) z=h(z)+u\left(z^{2}\right) k(z)=h_{0}(z)+h_{1}\left(z^{2}\right) z+u\left(z^{2}\right)\left\{\gamma\left(z^{2}\right)+\delta\left(z^{2}\right) z\right\}
$$

where we used the orthogonal splitting

$$
L^{2}(\mathbb{T})=S_{0} S_{0}^{*} L^{2}(\mathbb{T}) \oplus S_{1} S_{1}^{*} L^{2}(\mathbb{T})
$$

from Lemma 4.10. Similarly, from (6.5), we get

$$
\gamma\left(z^{2}\right)+\delta\left(z^{2}\right) z=k_{0}\left(z^{2}\right)+k_{1}\left(z^{2}\right) z
$$

and therefore $\gamma=k_{0}$ and $\delta=k_{1}$, by Lemma 5.1.

Collecting terms, and using the orthogonal splitting (6.6) we arrive at the following system of polynomial equations:

$$
\left\{\begin{array}{l}
\alpha=h_{0}+u \gamma \\
\beta=h_{1}+u \delta
\end{array}\right.
$$

or more precisely,

$$
\left\{\begin{array}{l}
\alpha(z)=h_{0}(z)+u(z) \gamma(z) \\
\beta(z)=h_{1}(z)+u(z) \delta(z)
\end{array}\right.
$$

It follows that the two functions $u, h$ may be determined from the Euclidean algorithm. With (6.3), we get

$$
\left\{\begin{array}{l}
u=q u o t(\gamma, \alpha) \\
h_{0}=\operatorname{rem}(\gamma, \alpha) \\
h_{1}=\operatorname{rem}(\delta, \beta)
\end{array}\right.
$$


Remark 6.1. The relevance of the determinant condition we have from Theorem 5.9 is as follows:

$$
\operatorname{det} A=\alpha \delta-\beta \gamma \equiv 1
$$

Substitution of (6.7) into this yields:

$$
h_{0} \delta-h_{1} \gamma \equiv 1 .
$$

Solutions to (6.7) are possible because the two polynomials $\delta(z)$ and $\gamma(z)$ are mutually prime. The derived matrix

$$
\left(\begin{array}{cc}
h_{0} & h_{1} \\
\gamma & \delta
\end{array}\right)
$$

is obtained from $A$ via a row-operation in the ring of polynomials.

For the inductive step, it is important to note:

$$
\operatorname{deg}\left(h_{0}\right)<\operatorname{deg}(\gamma) \text {, and } \operatorname{deg}\left(h_{1}\right)<\operatorname{deg}(\delta) .
$$

The next step, continuing from (6.4) is the determination of a matrix-function $C$, and three polynomials $p, q$, and $L$ such that

$$
\left(\begin{array}{cc}
1 & -U \\
0 & 1
\end{array}\right) A=\left(\begin{array}{ll}
1 & 0 \\
L & 1
\end{array}\right) C
$$

and

$$
\left(\begin{array}{cc}
1 & -U\left(z^{2}\right) \\
0 & 1
\end{array}\right) A\left(z^{2}\right)\left(\begin{array}{l}
1 \\
z
\end{array}\right)=\left(\begin{array}{cc}
1 & 0 \\
L\left(z^{2}\right) & 1
\end{array}\right)\left(\begin{array}{l}
p(z) \\
q(z)
\end{array}\right)
$$

Here

$$
\left(\begin{array}{l}
p \\
q
\end{array}\right)=C\left(z^{2}\right)\left(\begin{array}{l}
1 \\
z
\end{array}\right)
$$

The reader will notice that in this step, everything is as before with the only difference that now

$$
\left(\begin{array}{ll}
1 & 0 \\
L & 1
\end{array}\right)
$$

is lower diagonal in contrast with

$$
\left(\begin{array}{ll}
1 & U \\
0 & 1
\end{array}\right)
$$

in the previous step.

This time, the determination of the polynomial $p$ in (6.11) is automatic. With

$$
p(z)=p_{0}\left(z^{2}\right)+z p_{1}\left(z^{2}\right)
$$

(see (6.6)), and we get the following system:

$$
\begin{gathered}
\left\{\begin{array}{l}
p_{0}=\alpha-u \gamma=h_{0} \\
p_{1}=\beta-u \delta=h_{1} ; \quad \text { and }
\end{array}\right. \\
\left\{\begin{array}{l}
\gamma=L(\alpha-u \gamma)+q_{0}=L h_{0}+q_{0} \\
\delta=L(\beta-u \delta)+q_{1}=L h_{1}+q_{1}
\end{array}\right.
\end{gathered}
$$

So the determination of $L(z)$ and $q(z)=q_{0}\left(z^{2}\right)+z q_{1}\left(z^{2}\right)$ may be done with Euclid:

$$
\left\{\begin{array}{l}
L=q u o t(\alpha-u \gamma, \gamma)=q u o t\left(h_{0}, \gamma\right) \\
q_{0}=\operatorname{rem}(\alpha-u \gamma, \gamma)=\operatorname{rem}\left(h_{0}, \gamma\right) \\
q_{1}=\operatorname{rem}(\beta-u \delta, \delta)=\operatorname{rem}\left(h_{1}, \delta\right)
\end{array}\right.
$$


Combining the two steps, the comparison of degrees is as follows:

$$
\left\{\begin{array}{l}
\operatorname{deg}\left(q_{0}\right)<\operatorname{deg}\left(h_{0}\right)<\operatorname{deg}(\gamma) \\
\operatorname{deg}\left(q_{1}\right)<\operatorname{deg}\left(h_{1}\right)<\operatorname{deg}(\delta)
\end{array}\right.
$$

Two conclusions now follow:

(i) the procedure may continure by recursion;

(ii) the procedure must terminate.

Remark 6.2. In order to start the algorithm in (6.8) with direct reference to Euclid, we must have

$$
\operatorname{deg}(\gamma) \leq \operatorname{deg}(\alpha)
$$

where

$$
A=\left(\begin{array}{ll}
\alpha & \beta \\
\gamma & \delta
\end{array}\right)
$$

is the initial $2 \times 2$ matrix-function.

Now, suppose (6.14), i.e., that

$$
\operatorname{deg}(\gamma)>\operatorname{deg}(\alpha)
$$

Then determine a polynomial $L$ such that

$$
\operatorname{deg}(\gamma-L \alpha) \leq \operatorname{deg}(\alpha) .
$$

We may then start the procedure (6.8) on the matrix function

$$
\left(\begin{array}{cc}
\alpha & \beta \\
\gamma-L \alpha & \delta
\end{array}\right)=\left(\begin{array}{cc}
1 & 0 \\
-L & 1
\end{array}\right) A .
$$

If a polynomial $U$ and a matrix function $B$ is then found for

$$
\left(\begin{array}{cc}
\alpha & \beta \\
\gamma-L \alpha & \delta
\end{array}\right)
$$

then the factorization

$$
A=\left(\begin{array}{ll}
1 & 0 \\
L & 1
\end{array}\right)\left(\begin{array}{cc}
1 & U \\
0 & 1
\end{array}\right) B
$$

holds; and the recursion will then work as outlined.

In the following, starting with a matrix-function $A$, we will always assume that the degrees of the polynomials $\left(A_{i, j}\right)_{i, j \in \mathbb{Z}_{N}}$ have been adjusted this way, so the direct Euclidean algorithm can be applied.

6.2. The $3 \times 3$ case. The thrust of this section is the assertion that Theorem 5.9 holds with small modifications in the $3 \times 3$ case.

6.2.1. Comments: In the definition of $A \in S L_{3}(\mathrm{pol})$, it is understood that $A(z)$ has $\operatorname{det} A(z) \equiv 1$, and that the entries of the inverse matrix $A(z)^{-1}$ are again polynomials.

Note that if $L, M, U$ and $V$ are polynomials, then the four matrices

$$
\left(\begin{array}{ccc}
1 & 0 & 0 \\
L & 1 & 0 \\
0 & M & 1
\end{array}\right),\left(\begin{array}{ccc}
1 & 0 & 0 \\
0 & 1 & 0 \\
L & 0 & 1
\end{array}\right),\left(\begin{array}{ccc}
1 & U & 0 \\
0 & 1 & V \\
0 & 0 & 1
\end{array}\right) \text { and }\left(\begin{array}{ccc}
1 & 0 & U \\
0 & 1 & 0 \\
0 & 0 & 1
\end{array}\right)
$$

are in $S L_{3}(\mathrm{pol})$ since 


$$
\begin{gathered}
\left(\begin{array}{ccc}
1 & 0 & 0 \\
L & 1 & 0 \\
0 & M & 1
\end{array}\right)^{-1}=\left(\begin{array}{ccc}
1 & 0 & 0 \\
-L & 1 & 0 \\
L M & -M & 1
\end{array}\right), \text { and } \\
\left(\begin{array}{lll}
1 & U & 0 \\
0 & 1 & V \\
0 & 0 & 1
\end{array}\right)^{-1}=\left(\begin{array}{ccc}
1 & -U & U V \\
0 & 1 & -V \\
0 & 0 & 1
\end{array}\right)
\end{gathered}
$$

Theorem 6.3. Let $A \in S L_{3}(\mathrm{pol})$; then the conclusion in Theorem 5.9 carries over with the modification that the alternating upper and lower triangular matrixfunctions now have the form (6.16) or (6.17)-(6.18) where the functions $L_{j}, M_{j}, U_{j}$, and $V_{j}, j=1,2, \cdots$ are polynomials.

\subsection{The $N \times N$ case.}

Theorem 6.4. Let $N \in \mathbb{Z}_{+}, N>1$, be given and fixed. Let $A \in S L_{N}($ pol $)$; then the conclusions in Theorem 5.9 carry over with the modification that the alternative factors in the product are upper and lower triangular matrix-functions in $S L_{N}(\mathrm{pol})$. We may take the lower triangular matrix-factors of the form

$$
\left(\begin{array}{cccccccc}
1 & 0 & 0 & 0 & 0 & 0 & 0 & 0 \\
0 & 1 & 0 & 0 & 0 & 0 & 0 & 0 \\
L_{p} & 0 & 1 & 0 & 0 & 0 & 0 & 0 \\
0 & L_{p+1} & 0 & 1 & 0 & 0 & 0 & 0 \\
0 & 0 & . & 0 & 1 & 0 & 0 & 0 \\
0 & 0 & 0 & . & 0 & 1 & 0 & 0 \\
0 & 0 & 0 & 0 & . & 0 & 1 & 0 \\
0 & 0 & 0 & 0 & 0 & L_{N-1} & 0 & 1
\end{array}\right)
$$

$\mathcal{L}=\left(L_{i, j}\right)_{i, j \in \mathbb{Z}_{N}}$, polynomial entries

$$
\left\{\begin{array}{l}
L_{i, i} \equiv 1 \\
L_{i, j}(z)=\delta_{i-j, p} L_{i}(z)
\end{array}\right.
$$

and the upper triangular factors of the form $\mathcal{U}=\left(U_{i, j}\right)_{i, j \in \mathbb{Z}_{N}}$ with

$$
\left\{\begin{array}{l}
U_{i, i} \equiv 1, \\
L_{i, j}(z)=\delta_{i-j, p} U_{i}(z) ;
\end{array}\right.
$$

Proof. Notation. Let $U_{1}, \cdots, U_{N}, L_{1}, \cdots, L_{N}$ be polynomials, and set

$$
\mathcal{U}_{N}(U)=\left(\begin{array}{ccccccc}
1 & U_{1} & 0 & 0 & 0 & 0 & 0 \\
0 & 1 & U_{2} & 0 & 0 & 0 & 0 \\
0 & 0 & 1 & . & 0 & 0 & 0 \\
0 & 0 & 0 & 1 & . & 0 & 0 \\
0 & 0 & 0 & 0 & 1 & . & 0 \\
0 & 0 & 0 & 0 & 0 & 1 & U_{N-1} \\
0 & 0 & 0 & 0 & 0 & 0 & 1
\end{array}\right)
$$




$$
\mathcal{L}_{N}(L)=\left(\begin{array}{ccccccc}
1 & 0 & 0 & 0 & 0 & 0 & 0 \\
L_{1} & 1 & 0 & 0 & 0 & 0 & 0 \\
0 & L_{2} & 1 & 0 & 0 & 0 & 0 \\
0 & 0 & . & 1 & 0 & 0 & 0 \\
0 & 0 & 0 & . & 1 & 0 & 0 \\
0 & 0 & 0 & 0 & . & 1 & 0 \\
0 & 0 & 0 & 0 & 0 & L_{N-1} & 1
\end{array}\right)
$$

Note that both are in $S L_{N}(\mathrm{pol})$; and we have

$$
\begin{gathered}
\mathcal{U}_{N}(U)^{-1}=\mathcal{U}_{N}(-U), \quad \text { and } \\
\mathcal{L}_{N}(L)^{-1}=\mathcal{L}_{N}(-L) .
\end{gathered}
$$

Step 1: Starting with $A=\left(A_{i, j}\right) \in S L_{N}$ (pol). Then left-multiply with a suitably chosen $\mathcal{U}_{N}(-U)$ such that the degrees in the first column of $\mathcal{U}_{N}(-U) A$ decrease, i.e.,

$$
\operatorname{deg}\left(A_{0,0}\right) \leq \operatorname{deg}\left(A_{1,0}-u_{2} A_{1,0}\right) \leq \cdots \operatorname{deg}\left(A_{N-1,0}\right)
$$

In the following, we shall use the same letter $A$ for the modified matrix-function.

Step 2: Determine a system of polynomials $L_{1}, \cdots, L_{N-1}$ and a polynomial vector-function

such that

$$
\left[\begin{array}{c}
f_{0} \\
f_{1} \\
\cdots \\
f_{N-1}
\end{array}\right]
$$

$$
A_{N}\left[\begin{array}{c}
1 \\
z \\
z^{2} \\
\cdots \\
z^{N-1}
\end{array}\right]=\mathcal{L}_{N}(L)_{N}\left[\begin{array}{c}
f_{0} \\
f_{1} \\
\cdots \\
f_{N-1}
\end{array}\right]
$$

or equivalently

$$
\sum_{j=0}^{N-1} A_{i, j}\left(z^{N}\right) z^{j}=\left\{\begin{array}{ll}
f_{0}(z) & \text { if } i=0 \\
L_{i}\left(z^{N}\right) f_{i-1}(z)+f_{i}(z) & \text { if } i>0
\end{array} .\right.
$$

Step 3: Apply the operators $S_{j}$ and $S_{j}^{*}$ from (5.1) to both sides in (6.24). First (6.24) takes the form:

$$
\sum_{j=0}^{N-1} S_{j} A_{i, j}=\left\{\begin{array}{ll}
f_{0} & \text { if } i=0 \\
S_{f_{i-1}} L_{i}+f_{i} & \text { if } i>0
\end{array} .\right.
$$

For $i=1$, we get

$$
A_{1, j}=L_{1} A_{0, j}+k_{j} \quad \text { where } \quad k_{j}=S_{j}^{*} f_{1} .
$$

By (6.23) and the assumptions on the matrix-functions, we note that the system (6.25) may now be solved with the Euclidean algorithm:

$$
\left\{\begin{array}{l}
L_{1}=\operatorname{quot}\left(A_{0, j}, A_{1, j}\right) \\
k_{j}=\operatorname{rem}\left(A_{0, j}, A_{1, j}\right)
\end{array}\right.
$$


with the same polynomial $L_{1}$ for $j=0,1, \cdots, N-1$.

For the polynomial function $f_{1}$ we then have

$$
f_{1}=\sum_{j=0}^{N-1} S_{j} k_{j} ;
$$

i.e.

$$
f_{1}(z)=k_{0}\left(z^{N}\right)+k_{1}\left(z^{N}\right) z+\cdots+k_{N-1}\left(z^{N-1}\right) z^{N-1} .
$$

The process now continues recursively until all the functions $L_{1}, L_{2}, \cdots, f_{1}, f_{2}, \cdots$ have been determined.

Step 4: The formula (6.24) translates into a matrix-factorizations as follows: With $L$ and $F$ determined in (6.24), we get

$$
A=\mathcal{L}_{N}(L) B
$$

as a simple matrix-product taking $B=\left(B_{i, j}\right)$, and

$$
B_{i, j}=S_{j}^{*} f_{i}
$$

where we used Lemmas 4.10 and 5.1.

Step 5: The process now continues with the polynomial matrix-function from (6.28) and (6.29). We determine polynomials $U_{1}, \cdots, U_{N-1}$ and a third matrix function

$$
C=(C(z))=\left(C_{i, j}(z)\right) \text { such that } B=\mathcal{U}_{N}(U) C .
$$

Step 6: As each step of the process we alternate $L$ and $U$; and at each step, the degrees of the matrix-functions is decreased. Hence the recursion must terminate as stated in Theorem 6.4.

\section{QuAntization}

In addition to building algorithms for signal and image processing, there is the related problem of quantization. We define "quantization" broadly, and indeed there is a variety of approaches.

Indeed the "signals" may have a subtle form; the time variable might correspond to numbers in a system of pixel grids. The tools we developed in the previous sections are sufficiently versatile. For clarity of discussion, it helps to separate quantization of the two sides, input and output; so for example, "time" one and "magnitude" the other. The idea is to select a finite set of possibilities on either side, be it points, e.g., by sampling; or one might make suitable selections of intervals on the two sides of the quantization problem.

In order to adapt to hardware, and to reduce the number of computations, one makes a selection of a threshold. Specifically, when thresholding is applied to a set of numbers in an algorithm, the threshold function denoted $Q$ below) sends insignificant numbers (for example "very small") to zero.

In the thresholding process, applied to image processing, individual pixels are marked as object-pixels if their value is greater than some threshold value (assuming an object to be brighter than the background) and as background-pixels otherwise; a convention known as threshold above. This contrasts threshold below, or threshold inside, where a pixel is labeled "object" if its value is between two thresholds; and threshold outside; the opposite of threshold inside.

Below we will outline briefly recursive quantization schemes. The purpose is to illustrate how the particular filters we developed in section 5, and choice of 
threshold function, have the effect of making the recursive quantization schemes run faster, and be more effective. A popular method in recent papers (sigma delta quantization) is based on these ideas, plus the use of subtle difference/summation algorithms, see eg., [LPY10, LHR09]

The literature in the subject is vast. A pioneering paper [Ben48] opens up the door to the use of spectral analysis, and stochastic processes, especially amenable to the present results. On the theoretical side, recent papers are relevant: [Abd08, BOT08].

A key factor of the filtering algorithms from sections 4 and 6 is careful use of upsampling and downsampling. With a finite filter $\left(h_{1} h_{2}, \cdots\right)$, we get local input/output boxes (Fig. 4)

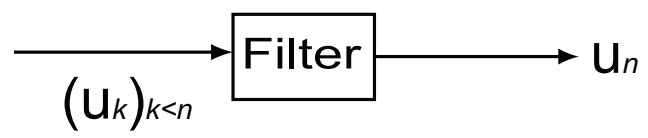

FiguRE 4. Standard filter.

where

$$
u_{n}=\sum_{j \geq 1} h_{j} u_{n-j}=h_{1} u_{n-1}+h_{2} u_{n-2}+\cdots
$$

or in matrix form

$$
\left(\begin{array}{ccccc}
0 & h_{1} & h_{2} & h_{3} & \cdots \\
0 & 0 & h_{1} & h_{2} & \cdots \\
\vdots & \vdots & \vdots & \vdots & \ddots
\end{array}\right) .
$$

For contrast, compare with the standard operator matrices from (5.6)

$$
\left(\begin{array}{cccccccc}
0 & 0 & h_{1} & h_{2} & h_{3} & h_{4} & h_{5} & \cdots \\
0 & 0 & 0 & 0 & h_{1} & h_{2} & h_{3} & \cdots \\
0 & 0 & 0 & 0 & 0 & 0 & h_{1} & \cdots \\
\vdots & \vdots & \vdots & \vdots & \vdots & \vdots & \vdots & \cdots \\
\vdots & \vdots & \vdots & \vdots & \vdots & \vdots & \vdots & \ddots
\end{array}\right) .
$$

For emphasis, we give (7.3) in diagram form

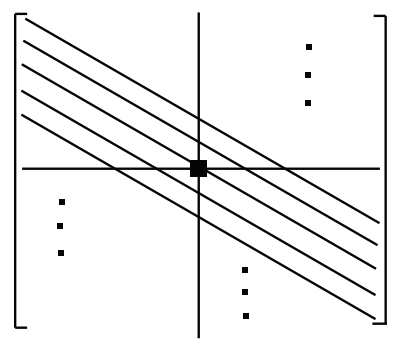

Figure 5. Filter operation with slanting. See Lemma 5.4. 
For the pyramid algorithm in Fig. 2, we use two versions of the slanted matrix in Fig. 5, high vs. low.

For the image processing (Fig. 3) we use four versions of the slanted matrices,

(a) a matrix that takes the average in horizontal direction

(b) a matrix that takes the average in vertical direction

(c) a matrix that takes the difference in horizontal direction

(d) a matrix that takes the difference in vertical direction.

which yield "average", "horizontal", "vertical" and "diagonal" details.

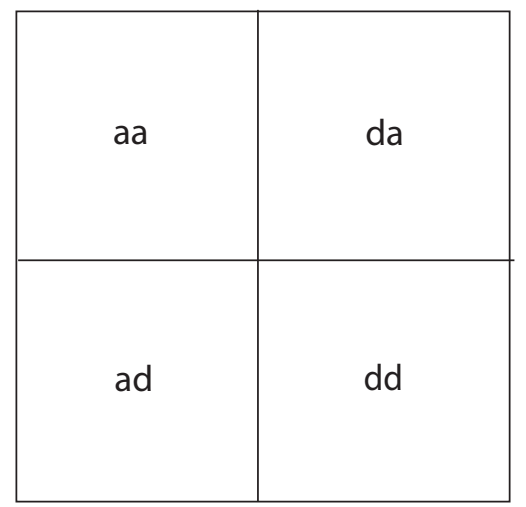

Figure 6. Level 1 decomposition. Clockwise: Average, horizontal, diagonal and vertical details.

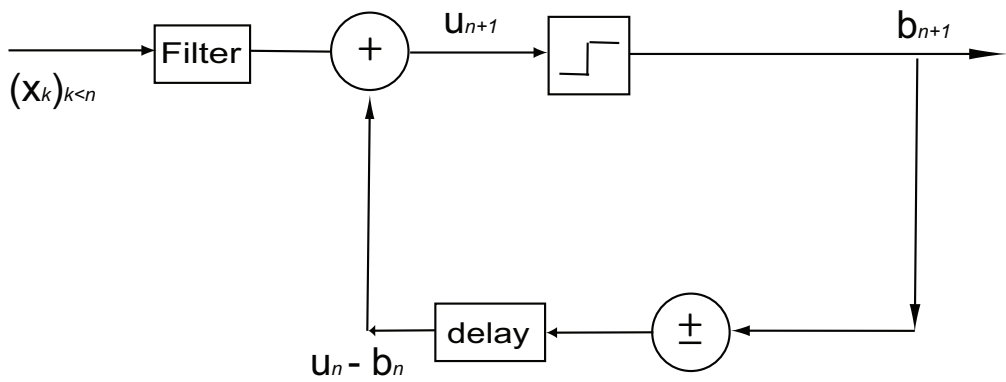

FiguRE 7. Quantization.

$$
\left\{\begin{array}{l}
u_{n+1}=(F u)_{n}+x_{n}-b_{n} \\
b_{n}=Q\left((F u)_{n}+x_{n}\right)
\end{array}\right.
$$

The figure and (7.4) together, thus summarizing the combined processes from the discussion and Fig 7:

The filter $F$ from the first eq in (7.4), and the first box in Fig 7, may be any one of those built in sections 4 through 6 above. So the particular filter $F$ selected may itself be the result of a factorization algorithm as outlined above: it may be a 
time series, a wireless signal, or a system of pixel values; and in each case, it may involve any number of frequency bands.

The output from $F$ (see Fig 7 ) will pass through a thresholding filter $Q$, thus outputting $b_{n+1}$. In symbols, the next two steps are: "take difference", and timeshift the result ("delay"), so from $n+1$ back to $n$. The first eq in (7.4) indicates how the process repeats itself, but with the output from the previous step, as input in the next.

\section{REFERENCES}

[Abd08] Fatma Abdelkefi. Performance of sigma-delta quantizations in finite frames. IEEE Trans. Inform. Theory, 54(11):5087-5101, 2008.

[Akh65] N. I. Akhiezer. The classical moment problem and some related questions in analysis. Translated by N. Kemmer. Hafner Publishing Co., New York, 1965.

[Ben48] W. R. Bennett. Spectra of quantized signals. Bell System Tech. J., 27:446-472, 1948.

[BJ02a] Ola Bratteli and Palle Jorgensen. Wavelets through a looking glass. Applied and Numerical Harmonic Analysis. Birkhäuser Boston Inc., Boston, MA, 2002. The world of the spectrum.

[BJ02b] Ola Bratteli and Palle E. T. Jorgensen. Wavelet filters and infinite-dimensional unitary groups. In Wavelet analysis and applications (Guangzhou, 1999), volume 25 of AMS/IP Stud. Adv. Math., pages 35-65. Amer. Math. Soc., Providence, RI, 2002.

[BOT08] John J. Benedetto, Onur Oktay, and Aram Tangboondouangjit. Complex sigma-delta quantization algorithms for finite frames. In Radon transforms, geometry, and wavelets, volume 464 of Contemp. Math., pages 27-49. Amer. Math. Soc., Providence, RI, 2008.

[BRa] Chris Brislawn and I. G. Rosen. Group lifting structures for multirate filter banks, i: Uniqueness of lifting factorizations.

[BRb] Chris Brislawn and I. G. Rosen. Group lifting structures for multirate filter banks, ii: Uniqueness of lifting factorizations.

[BR91] Chris Brislawn and I. G. Rosen. Wavelet based approximation in the optimal control of distributed parameter systems. Numer. Funct. Anal. Optim., 12(1-2):33-77, 1991.

[CC08] X. X. Chen and Y. Y. Chen. Self-lifting scheme: new approach for generating and factoring wavelet filter bank. IET Signal Process., 2(4):405-414, 2008.

[DS98] Ingrid Daubechies and Wim Sweldens. Factoring wavelet transforms into lifting steps. J. Fourier Anal. Appl., 4(3):247-269, 1998.

[HCXH08] Yumin He, Xuefeng Chen, Jiawei Xiang, and Zhengjia He. Multiresolution analysis for finite element method using interpolating wavelet and lifting scheme. Comm. Numer. Methods Engrg., 24(11):1045-1066, 2008.

[Iwa49] Kenkichi Iwasawa. On some types of topological groups. Ann. of Math. (2), 50:507$558,1949$.

[JlCH01] A. Jensen and A. la Cour-Harbo. Ripples in mathematics. Springer-Verlag, Berlin, 2001. The discrete wavelet transform.

[JS09] Palle E. T. Jorgensen and Myung-Sin Song. Analysis of fractals, image compression, entropy encoding, Karhunen-Loève transforms. Acta Appl. Math., 108(3):489-508, 2009.

[Law99] Wayne M. Lawton. Conjugate quadrature filters. In Advances in wavelets (Hong Kong, 1997), pages 103-119. Springer, Singapore, 1999.

[Law00] Wayne Lawton. Infinite convolution products and refinable distributions on Lie groups. Trans. Amer. Math. Soc., 352(6):2913-2936, 2000.

[Law02] Wayne Lawton. Global analysis of wavelet methods for Euler's equation. Mat. Model., 14(5):75-88, 2002. Second International Conference OFEA'2001 "Optimization of Finite Element Approximation, Splines and Wavelets" (Russian) (St. Petersburg, 2001).

[Law04] Wayne M. Lawton. Hermite interpolation in loop groups and conjugate quadrature filter approximation. Acta Appl. Math., 84(3):315-349, 2004.

[LHR09] B. W. K. Ling, C. Y. F. Ho, and J. D. Reiss. Control of sigma delta modulators via fuzzy impulsive approach. In Control of chaos in nonlinear circuits and systems, volume 64 of World Sci. Ser. Nonlinear Sci. Ser. A Monogr. Treatises, pages 245-270. World Sci. Publ., Hackensack, NJ, 2009. 
[LPY10] M Lammers, A. M. Powell, and Özgür Yılmaz. Alternative dual frames for digitalto-analog conversion in sigma-delta quantization. Adv. Comput. Math., 32(1):73-102, 2010.

[SBT03] Peng-Lang Shui, Zheng Bao, and Yuan Yan Tang. Three-band biorthogonal interpolating complex wavelets with stopband suppression via lifting scheme. IEEE Trans. Signal Process., 51(5):1293-1305, 2003.

[Son06] Myung-Sin Song. Wavelet image compression. In Operator theory, operator algebras, and applications, volume 414 of Contemp. Math., pages 41-73. Amer. Math. Soc., Providence, RI, 2006.

[SR91] Wim Sweldens and Dirk Roose. Shape from shading using parallel multigrid relaxation. In Multigrid methods, III (Bonn, 1990), volume 98 of Internat. Ser. Numer. Math., pages 353-364. Birkhäuser, Basel, 1991.

[Swe96] Wim Sweldens. The lifting scheme: a custom-design construction of biorthogonal wavelets. Appl. Comput. Harmon. Anal., 3(2):186-200, 1996.

[Swe98] Wim Sweldens. The lifting scheme: a construction of second generation wavelets. SIAM J. Math. Anal., 29(2):511-546 (electronic), 1998.

Department of Mathematics, The University of Iowa, Iowa City, IA52242, USA

E-mail address: jorgen@math.uiowa.edu

$U R L:$ http://www. math.uiowa.edu/ ${ }^{\text {jorgen }}$

Department of Mathematics and Statistics, Southern Illinois University Edwardsville, EDWARDSVILLE, IL62026, USA

URL: http://www.siue.edu/ msong 